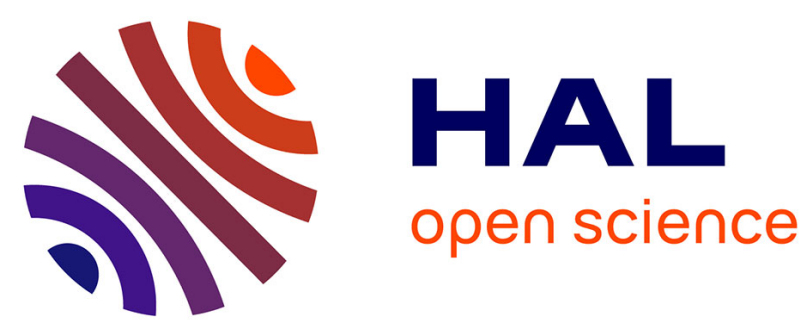

\title{
Non-linéarités d'absorption intrabande dans les puits quantiques GaAs-AlxGa1-xAs
}

F.H. Julien, J.-M. Lourtioz, N. Herschkorn, D. Delacourt, J.P. Pocholle, M. Papuchon, R. Planel, G. Le Roux

\section{- To cite this version:}

F.H. Julien, J.-M. Lourtioz, N. Herschkorn, D. Delacourt, J.P. Pocholle, et al.. Non-linéarités d'absorption intrabande dans les puits quantiques GaAs-AlxGa1-xAs. Revue de Physique Appliquée, 1989, 24 (1), pp.37-43. 10.1051/rphysap:0198900240103700 . jpa-00246026

\section{HAL Id: jpa-00246026 https://hal.science/jpa-00246026}

Submitted on 1 Jan 1989

HAL is a multi-disciplinary open access archive for the deposit and dissemination of scientific research documents, whether they are published or not. The documents may come from teaching and research institutions in France or abroad, or from public or private research centers.
L'archive ouverte pluridisciplinaire HAL, est destinée au dépôt et à la diffusion de documents scientifiques de niveau recherche, publiés ou non, émanant des établissements d'enseignement et de recherche français ou étrangers, des laboratoires publics ou privés. 


\title{
Non-linéarités d'absorption intrabande dans les puits quantiques GaAs- $\mathbf{A l}_{x} \mathbf{G a}_{1-x} \mathbf{A s}$
}

\author{
F. H. Julien $\left({ }^{1}\right)$, J.-M. Lourtioz $\left({ }^{1}\right)$, N. Herschkorn $\left({ }^{2}\right)$, D. Delacourt $\left({ }^{2}\right)$, \\ J. P. Pocholle $\left({ }^{2}\right)$, M. Papuchon $\left({ }^{2}\right)$, R. Planel $\left({ }^{3}\right)$ et G. Le Roux $\left({ }^{3}\right)$ \\ (') Institut d'Electronique Fondamentale, U.A. 22 du CNRS, Université Paris XI, 91405 Orsay, France \\ ( $\left.{ }^{2}\right)$ Laboratoire Central de Recherches, Thomson-CSF, 91401 Orsay, France \\ ( $\left.{ }^{3}\right)$ Groupement Scientifique CNET-CNRS, U.P. 20 du CNRS, 196 Avenue H. Ravera, 92220 Bagneux, \\ France
}

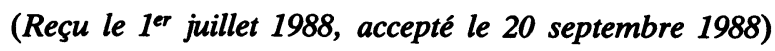

\begin{abstract}
Résumé. - Les transitions optiques entre niveaux de la bande de conduction des puits quantiques font l'objet d'études approfondies pour le développement de photodétecteurs infrarouges rapides et de nouveaux dispositifs opto-électroniques. La non-linéarité liée à la saturation de la transition d'absorption intrabande est démontrée. L'étude nous permet d'identifier les mécanismes de relaxation intrabande.
\end{abstract}

\begin{abstract}
Optical transitions between confined energy levels of the conduction band of quantum well structures are of great interest for the development of fast infrared detectors and new optoelectronic devices. The non-linearity due to the intersubband absorption saturation is investigated. The intersubband relaxation processes are identified.
\end{abstract}

\section{Introduction.}

Une transition intrabande dans la bande de conduction d'un puits quantique implique l'absorption d'un photon infrarouge provoquant l'excitation d'un électron d'un niveau confiné $n$ du puits quantique à un niveau $m$ d'énergie supérieure. Contrairement aux transitions interbandes, les transitions intrabandes permises dans un puits symétrique correspondent à $\Delta n$ impair. De plus, seule une onde optique polarisée perpendiculairement aux plans des couches du puits quantique peut induire une transition d'absorption intrabande. Ces transitions ont pour la première fois été mises en évidence par Pinczuk et al. [1-3] grâce à des techniques de diffusion inélastique. West et Eglash [4] ont observé directement l'absorption intrabande dans la bande de conduction d'un puits quantique multiple AlGaAs. Ces auteurs ont montré que les forces de transition associées sont considérables $(\sim 14)$. Les moments dipolaires sont typiquement de 10 à 30 e Å. La possibilité d'une émission stimulée intrabande a été étudiée par Kazarinov et Suris [5] mais n'a pas encore reçu de confirmation expérimentale. La modulation de la fréquence de la transition intrabande par effet Stark a été démontrée par Harwit et Harris [6]. Très récemment, ces propriétés remarquables sur un domaine étendu de longueurs d'ondes infrarouges $(2,5 \mu \mathrm{m}$ à $200 \mu \mathrm{m})$ ont motivé un grand nombre de travaux en vue de développer des photodétecteurs rapides infrarouges ainsi que des modulateurs de phase ou d'amplitude [7-10]. Signalons la mise en évidence d'un effet Stark optique sur une transition interbande induit par une onde infrarouge en proche résonance avec une transition d'absorption intrabande [11], ouvrant ainsi de nouvelles perspectives pour le développement de modulateurs tout-optique proche-infrarouge.

L'objet de cette étude est de déterminer le seuil de non-linéarité de l'absorption intrabande. Il s'agit d'une donnée cruciale non seulement pour les performances des photodétecteurs intrabandes mais aussi pour le développement à terme de nouveaux dispositifs non linéaires. Nous montrons que l'intensité de saturation est de l'ordre de $300 \mathrm{~kW} / \mathrm{cm}^{2}$, valeur d'intensité tout à fait accessible dans des structures à guide d'ondes. Outre le fait qu'il s'agit d'une détermination directe, l'originalité de l'étude est qu'elle a été conduite sur deux types de structures à puits quantiques à dopage modulé et anti-modulé. La détermination de l'intensité de saturation de la transition d'absorption intrabande permet de remonter au temps de vie des niveaux de la bande de conduction des puits quantiques. Les temps de vie trouvés de l'ordre de $10 \mathrm{ps}$ sont caractéristiques d'une relaxation par phonons optiques longitudinaux. 


\section{Echantillons.}

Les deux types d'échantillons ont été réalisés par épitaxie par jets moléculaires. Les puits quantiques sont dopés $n$ avec du silicium soit dans les barrières AlGaAs pour l'échantillon A soit dans les puits pour l'échantillon $\mathrm{B}$, de manière à assurer un peuplement du premier niveau de la bande de conduction. Du fait de l'accumulation de charges due au dopage, le profil de bande de l'échantillon A s'apparente à celui d'une structure à dopage modulé tandis que celui de l'échantillon $\mathrm{B}$ correspond à une structure à dopage anti-modulé.

L'échantillon A comprend un substrat GaAs semiisolant, une couche GaAs de $5000 \AA$ dopée $n^{+}$, suivi des 150 puits quantiques, puis d'une couche $\mathrm{Al}_{0,3} \mathrm{Ga}_{0,7} \mathrm{As}$ de $1200 \AA$ et d'une couche GaAs de $5000 \AA$ dopée $n^{+}$. Les puits quantiques se composent d'un puits GaAs non dopé de largeur $85 \AA$ et d'une barrière $\mathrm{Al}_{0,3} \mathrm{Ga}_{0,7} \mathrm{As}$ de $296 \AA$. Pour chaque barrière, seule une portion centrale de largeur $240 \AA$ est dopée $n$ à $5,5 \pm 1,5 \times 10^{17} \mathrm{~cm}^{-3}$. L'échantillon $\mathrm{B}$ comprend un substrat semi-isolant suivi d'une couche GaAs non dopée de $5000 \AA$, des 110 puits quantiques et d'une couche GaAs non dopée de $100 \AA$. Les puits quantiques multiples se composent de puits GaAs de largeur $96 \AA$ et de barrières $\mathrm{Al}_{0,57} \mathrm{Ga}_{0,43} \mathrm{As}$ de même largeur. Des 110 puits quantiques, seuls les derniers 100 puits sont dopés $n$ à $1,8 \times 10^{18} \mathrm{~cm}^{-3}$. Chaque échantillon est poli optiquement côté substrat.

Pour l'échantillon A, l'épaisseur de chaque couche a été contrôlée in situ à l'aide de la technique RHEED (reflection high-energy electron diffraction). Une technique de double diffraction $\mathrm{X}$ a été utilisée a posteriori pour déterminer la périodicité des puits quantiques multiples $(192 \AA)$ et la teneur moyenne en $\mathrm{Al}$ de l'échantillon $\mathrm{B}$. Seuls des pics de diffraction d'ordres impairs apparaissent sur le spectre $X$, ce qui est caractéristique d'une structure où la barrière et le puits ont la même épaisseur. De plus, la finesse des pics montrent qu'un excellent contrôle des épaisseurs a été obtenu tout au long de la croissance de l'échantillon B. La densité de porteurs dans les puits quantiques a été déterminée par une mesure de Hall. Elle s'élève à 1,32 $0,36 \times 10^{12} \mathrm{~cm}^{-2}$ pour l'échantillon $\mathrm{A}$ et $1,9 \times 10^{12} \mathrm{~cm}^{-2}$ pour l'échantillon $B$.

\section{Caractérisation en petit signal.}

Toutes les expériences ont été conduites en plaçant les échantillons à l'angle de Brewster $\left(74^{\circ}\right)$. La figure 1 montre que cette configuration conduit à un faible couplage de l'onde optique incidente polarisée avec la transition d'absorption intrabande du fait des règles de sélection sur la polarisation du champ électrique. Cet effet est partiellement compensé par

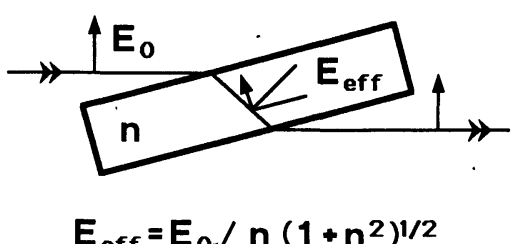

Fig. 1. - Seule la composante de polarisation de l'onde optique incidente perpendiculaire aux plans des couches des puits quantiques induit une absorption intrabande. A l'angle de Brewster, $E_{\text {eff }} / E_{0}$ n'est que de $9 \%$.

[Only radiation with electric field vector perpendicular to the layer planes experiences intersubband absorption. With a sample oriented at Brewster's angle, $E_{\text {eff }} / E_{0}$ is only $9 \%$.]

le grand nombre de puits quantiques présents dans les échantillons.

Les mesures d'absorption non saturée ont été menées à température ambiante à l'aide d'un interféromètre infrarouge Perkin-Elmer et en lumière polarisée. Les figures $2 a$ et $2 b$ montrent le spectre d'absorbance ( $-\log (T)$, où $T$ est la transmission de l'échantillon) des échantillons $\mathrm{A}$ et $\mathrm{B}$ en fonction de la longueur d'onde ou de l'énergie des photons en $\mathrm{cm}^{-1}$. Le spectre de l'échantillon $\mathrm{A}$ est corrigé de l'absorption par les porteurs libres provenant de la présence des deux couches GaAs fortement dopées. Le pic d'absorption est attribué à la transition d'absorption intrabande car il disparaît lorsque la source lumineuse est polarisée parallèlement aux couches des puits quantiques multiples. La résonance intrabande entre le niveau fondamental des puits quantiques et le premier niveau excité se produit à $10,45 \mu \mathrm{m}$ (119 meV) pour l'échantillon $A$ et $10,15 \mu \mathrm{m}$ (122 meV) pour l'échantillon $B$. La largeur totale à mi-hauteur est de l'ordre de $20 \mathrm{meV}$. L'absorbance s'élève à 0,53 pour l'échantillon $\mathrm{A}$ et 0,27 pour l'échantillon $\mathrm{B}$.

Il est intéressant de déduire à partir de ces valeurs d'absorbance, le coefficient d'absorption $\alpha_{0}$ par puits quantique, qui est caractéristique du matériau :

$$
\alpha_{0}=\frac{A_{0}}{L_{\mathrm{p}} N_{\mathrm{p}}} \frac{n}{\sqrt{n^{2}+1}}
$$

où $A_{0}$ est l'absorbance mesurée, $L_{\mathrm{p}}$ est la largeur du puits quantique, $N_{\mathrm{p}}$ est le nombre effectif de puits contribuant à l'absorption et $n \approx 3,2$ l'indice de réfraction du matériau. Avec les valeurs données plus haut, $\alpha_{0} \approx 4190 \mathrm{~cm}^{-1}$ pour l'échantillon $A$ et $\alpha_{0} \approx 2920 \mathrm{~cm}^{-1}$ pour l'échantillon $B$.

La position en énergie des niveaux du puits quantique a été estimée à partir d'un modèle de puits rectangulaire prenant en compte les paramètres de croissance des échantillons et les non-parabolicités de bande $[12,13]$. Le modèle prédit des résonan- 

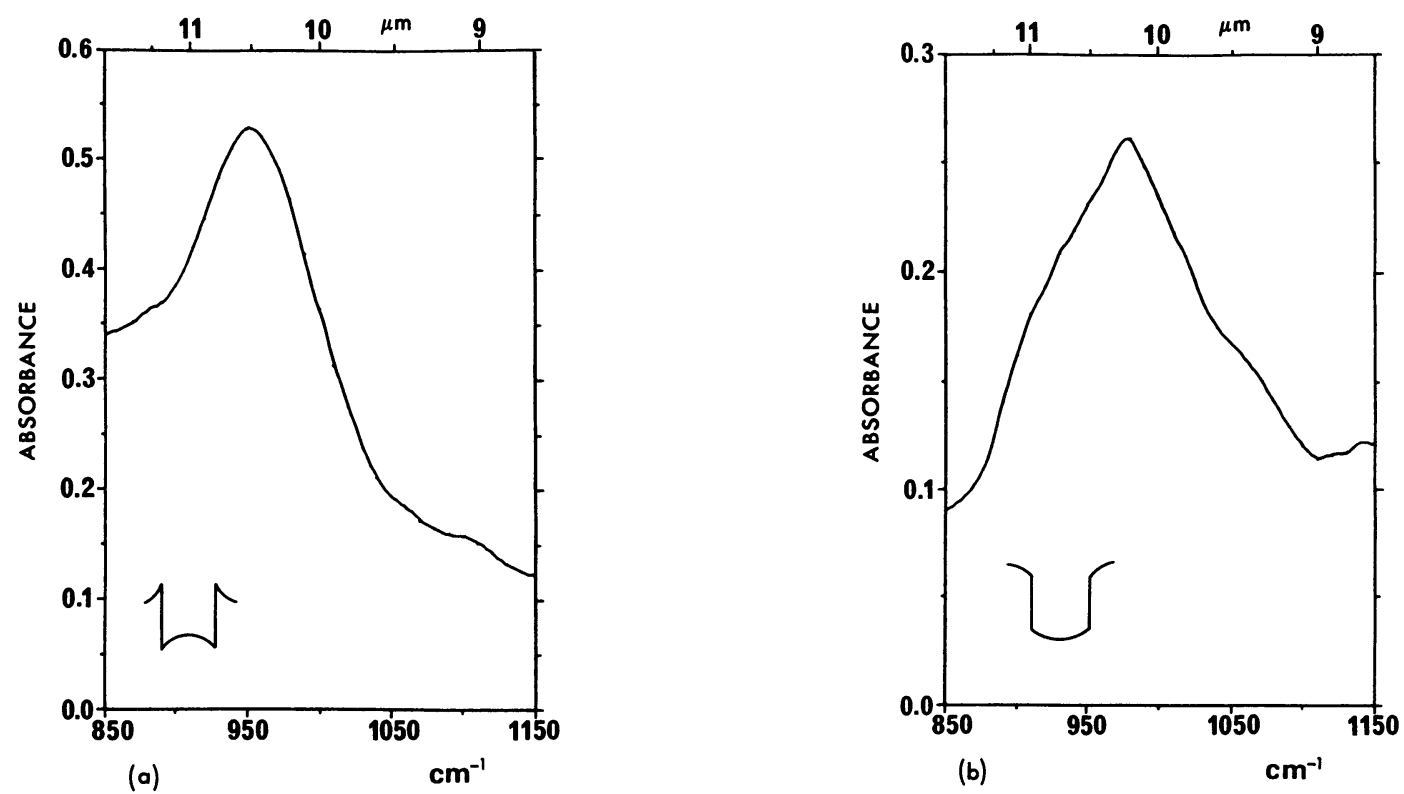

Fig. 2. - Absorbance en petit signal des échantillons en fonction de l'énergie des photons en $\mathrm{cm}^{-1}$ ou de la longueur d'onde en $\mu \mathrm{m}$; (a) et (b) correspondent respectivement aux échantillons $\mathrm{A}$ et $\mathrm{B}$.

[Small-signal absorbance as a function of the photon energy in $\mathrm{cm}^{-1}$ or the photon wavelength in $\mu \mathrm{m}$; (a) and (b) refer to sample $A$ and sample $B$ respectively.]

ces intrabandes à plus faible énergie : $109 \mathrm{meV}$ (A) et $106 \mathrm{meV}$ (B). L'introduction d'un potentiel de Hartree pour simuler le profil de bande conduit à une énergie des résonances encore inférieure d'environ $10 \mathrm{meV}$ avec les densités de porteurs existant dans les échantillons [14]. Un accord théorie-expérience plus satisfaisant serait probablement obtenu si la renormalisation de la bande de conduction était prise en compte dans le modèle.

\section{Configuration expérimentale en fort signal.}

La figure 3 montre le schéma expérimental utilisé pour caractériser la saturation de l'absorption intrabande. La source infrarouge est un laser $\mathrm{CO}_{2}$ TEA rendu monofréquence par injection du faisceau d'un laser $\mathrm{CO}_{2}$ continu. Le laser qui délivre $15 \mathrm{~mJ}$ en $110 \mathrm{~ns}$, est accordé pour chaque échantillon au centre de la résonance intrabande. Le diamètre de

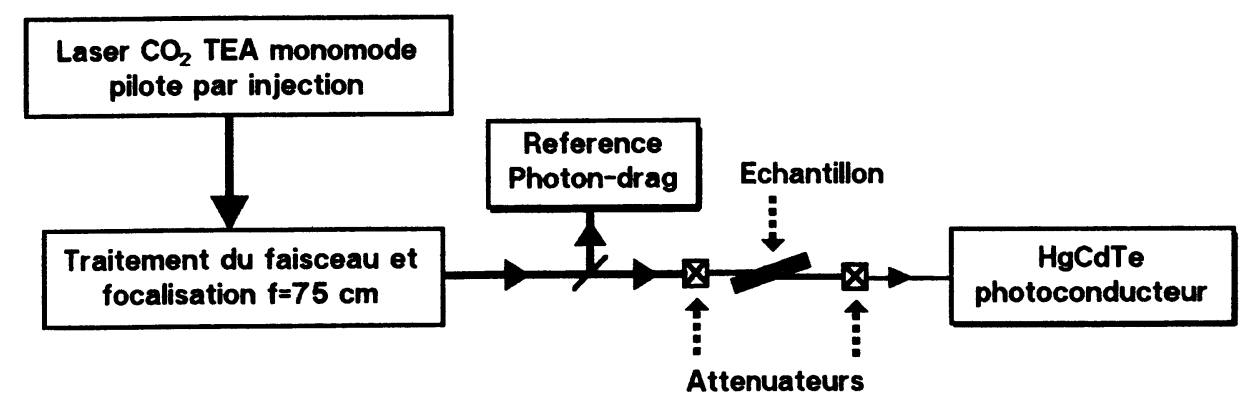

Fig. 3. - Schéma expérimental utilisé pour caractériser les non-linéarités d'absorption intrabande. Le laser $\mathrm{CO}_{2}$ TEA et son laser $\mathrm{CO}_{2}$ d'injection sont accordés sur la raie 10P6 pour l'échantillon A et 10R32 pour l'échantillon B. Une partie du faisceau infrarouge incident est détectée par un détecteur photon-drag de $1 \mathrm{~ns}$ de temps de montée. Le temps de montée du détecteur $\mathrm{HgCdTe}$ photoconducteur utilisé pour le signal transmis est de $0,7 \mathrm{~ns}$. Lors des expériences, la stabilité en puissance du laser est contrôlée par le détecteur photon-drag. L'intensité incidente sur l'échantillon n'est variée qu'en jouant sur le nombre d'atténuateurs placés avant l'échantillon. Le nombre total d'atténuateurs placés avant et après l'échantillon est toujours maintenu constant.

[Experimental set-up used to characterize the intersubband absorption non-linearity. The $\mathrm{CO}_{2}$ TEA laser and the $\mathrm{CO}_{2}$ injection laser are either tuned on the 10P6 line (sample A) or the 10R32 line (sample B). A photon-drag detector with $1 \mathrm{~ns}$ rise-time is used to control the laser stability. The transmitted signal is recorded by a $\mathrm{HgCdTe}$ photoconductor detector with $0.7 \mathrm{~ns}$ rise-time. During the experiments, the input intensity is only varied by changing the number of attenuators ahead of the sample. The overall number of attenuators is always kept constant.] 
pincement du faisceau infrarouge focalisé au niveau de l'échantillon est $2 w_{0}=0,46 \pm 0,04 \mathrm{~mm}$. Le système de détection rapide fait appel à un détecteur photon-drag pour le faisceau incident et à un détecteur $\mathrm{HgCdTe}$ photoconducteur pour le faisceau transmis. Des atténuateurs calibrés en $\mathrm{CaF}_{2}$ sont utilisés pour varier l'intensité infrarouge incidente sur l'échantillon mais aussi pour faire fonctionner le détecteur $\mathrm{HgCdTe}$ en régime linéaire. La transmission de l'échantillon est déduite de l'enregistrement du signal $\mathrm{HgCdTe}$ sur un oscilloscope rapide à mémoire, comme l'illustre la figure 4.

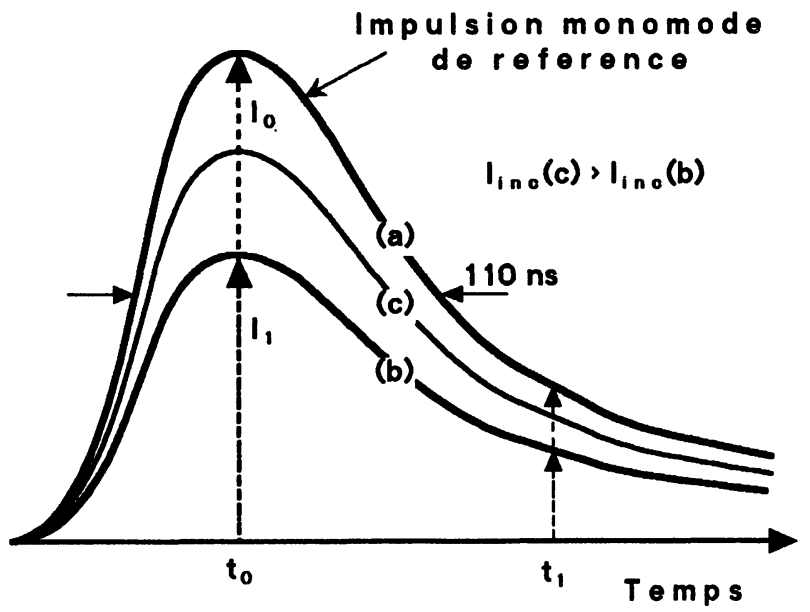

Fig. 4. - Exemple d'enregistrement des impulsions transmises permettant la détermination de la transmission de l'échantillon. L'impulsion (a) est l'impulsion du laser $\mathrm{CO}_{2}$ monomode transmise sans échantillon. Les impulsions (b) et (c) sont obtenues avec l'échantillon dans sa monture. Pour l'impulsion (c), l'intensité incidente sur l'échantillon est supérieure à celle correspondant à l'impulsion (b). La saturation de l'absorption intrabande est évidente. La transmission de l'échantillon au maximum de l'impulsion est donnée par le rapport des amplitudes $I_{1} / I_{0}$. Des mesures complémentaires sont obtenues à différents instants de l'impulsion.

[Determination of the sample transmission. Curve (a) is the transmitted $\mathrm{CO}_{2}$ laser pulse with no sample. Curves (b) and (c) are similar to curve (a) but with the sample mounted. For pulse (c), incident intensity is larger than for pulse (b). Intersubband absorption saturation is clear. The sample transmission at pulse maximum is given by the amplitude ratio $I_{1} / I_{0}$. Supplementary data points are obtained at various time delays after trigger.]

\section{Absorption non linéaire.}

Les figures $5 \mathrm{a}$ et $5 \mathrm{~b}$ montrent l'absorbance des échantillons $\mathrm{A}$ et $\mathrm{B}$ en fonction de l'intensité effective $I_{0}$ dans l'échantillon [4] :

$$
I_{0}=\frac{I_{\text {inc }}}{n\left(1+n^{2}\right)}
$$

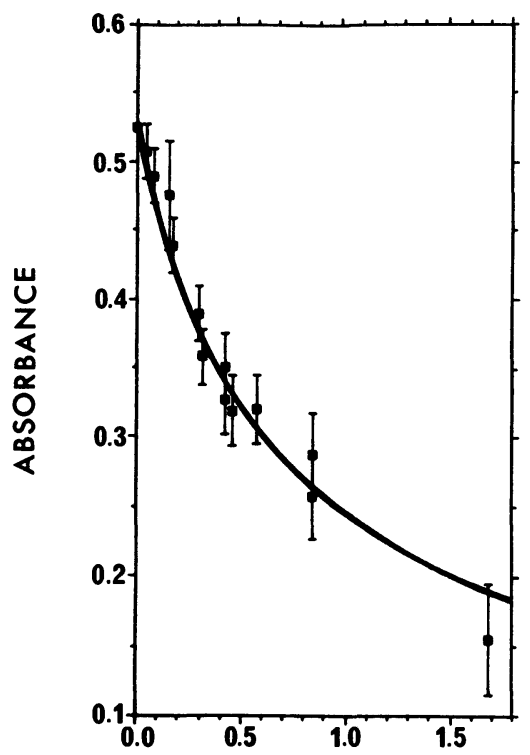

(a)

INTENSITE $\left(\mathrm{MW} / \mathrm{cm}^{2}\right)$

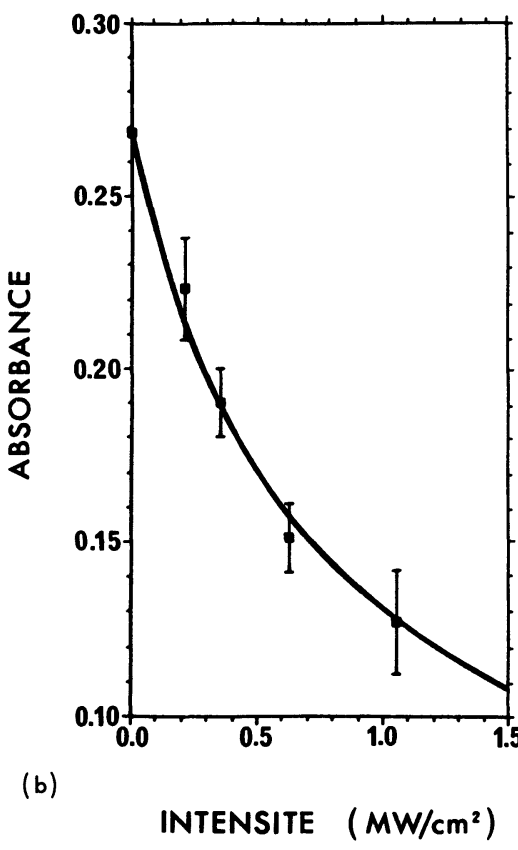

Fig. 5. - Absorbance des échantillons en fonction de l'intensité effective dans l'échantillon. (a) et (b) correspondent respectivement aux échantillons A et B. Les points correspondent à l'absorbance mesurée au maximum de l'impulsion. Les courbes en trait plein sont le résultat d'un modèle de saturation où l'intensité de saturation $I_{\mathrm{S}}$ est le seul paramètre ajustable ; a) $I_{\mathrm{S}}=340 \mathrm{~kW} / \mathrm{cm}^{2}$, b) $I_{\mathrm{S}}=$ $375 \mathrm{~kW} / \mathrm{cm}^{2}$.

[Absorbance of the sample as a function of the effective infrared intensity inside the sample; (a) and (b) refer to sample $A$ and sample $B$ respectively. The dots are experiments. The solid lines are theoretical fits using a saturation intensity value of $340 \mathrm{~kW} / \mathrm{cm}^{2}$ for sample $A$ and of $375 \mathrm{~kW} / \mathrm{cm}^{2}$ for sample B.] 
où $I_{\text {inc }}$ est l'intensité du faisceau infrarouge incident au niveau de l'échantillon. Les points expérimentaux sur les figures 4 donnent l'absorbance mesurée au maximum de l'impulsion laser. Les courbes sont le résultat d'un modèle et seront décrites plus loin. La décroissance de l'absorbance de l'échantillon avec l'accroissement de l'intensité incidente est caractéristique d'un milieu absorbant saturable. L'enregistrement des données est limité à $I_{0}=1,7 \mathrm{MW} / \mathrm{cm}^{2}$ ( $\left(I_{\text {inc }} \simeq 60 \mathrm{MW} / \mathrm{cm}^{2}\right)$ à cause de l'apparition d'un claquage plasma à la surface de l'échantillon. Pour chaque impulsion laser, des points expérimentaux supplémentaires ont été obtenus en mesurant la transmission de l'échantillon à différents instants après le déclenchement de l'impulsion. Il n'apparaît pas de différence significative avec les résultats rapportés sur les figures 5 . Ceci suggère déjà que l'élévation de température de l'échantillon durant l'impulsion laser reste modérée. De plus, les effets à $n$-corps [15] ou les effets de dépolarisation [16] qui modifieraient l'énergie de la résonance intrabande avec l'intensité excitatrice, semblent jouer un rôle mineur dans nos conditions expérimentales.

\section{Discussion.}

Pour estimer l'intensité de saturation de la transition d'absorption intrabande, nous avons supposé en première approximation que le système en interaction pouvait se limiter aux deux premiers niveaux du puits quantique, les relaxations s'effectuant uniquement entre les deux niveaux. En tenant compte du profil gaussien du faisceau incident, la variation de l'absorbance en fonction de l'intensité effective dans l'échantillon peut alors être décrite par :

$$
A\left(I_{0}\right)=A_{0} \frac{I_{\mathrm{S}}}{I_{0}} \operatorname{Ln}\left[1+\frac{I_{0}}{I_{\mathrm{S}}}\right]
$$

où $A_{0}$ est l'absorbance en petit signal et $I_{\mathrm{S}}$ l'intensité de saturation à déterminer. Les courbes des figures 5 montrent l'évolution de l'absorbance donnée par la formule précédente en prenant $I_{\mathrm{S}}=340 \mathrm{~kW} / \mathrm{cm}^{2}$ (échantillon A) et $I_{\mathrm{S}}=375 \mathrm{~kW} / \mathrm{cm}^{2}$ (échantillon B). L'accord avec les points expérimentaux est remarquable. Si l'on tient compte de l'incertitude sur les paramètres expérimentaux, l'intensité de saturation est $I_{\mathrm{S}}=340 \pm 120 \mathrm{~kW} / \mathrm{cm}^{2}$ pour l'échantillon A et $I_{\mathrm{S}}=375 \pm 120 \mathrm{~kW} / \mathrm{cm}^{2}$ pour l'échantillon $\mathrm{B}$. Ces valeurs d'intensité de saturation sont bien inférieures à celles calculées en référence [17] à partir d'un traitement par la matrice densité. Nous pensons que les temps de relaxation introduits dans ce modèle sont tout à fait irréalistes.

Le temps de vie des porteurs dans le deuxième niveau du puits quantique peut maintenant être estimé à partir des valeurs d'intensité de saturation :

$$
T_{1}=\frac{E}{2 \sigma I_{\mathrm{S}}}
$$

où $E$ est l'énergie de la transition intrabande et $\sigma$ est la section efficace d'absorption, que l'on peut déduire de la valeur d'absorbance en petit signal $A_{0}$ :

$$
\sigma \simeq \frac{0,956 A_{0}}{N_{\mathrm{p}} n_{\mathrm{s}}}
$$

où $N_{\mathrm{p}}$ est le nombre effectif de puits quantiques contribuant à l'absorption, $n_{\mathrm{s}}$ est la densité surfacique de porteurs dans chaque puits, et le facteur numérique est une correction géométrique due à l'angle de Brewster. Avec les valeurs données plus haut, le temps de vie des niveaux est de $T_{1} \simeq$ $10,6 \pm 3,5 \mathrm{ps}$ pour l'échantillon $\mathrm{A}$ et $T_{1} \simeq$ $15,5 \pm 5$ ps pour l'échantillon $B$. Ces valeurs sont comparables avec les temps de vie de l'ordre de 14 ps et 11 ps mesurés pour des puits de largeur 47 et $51 \AA$ en référence [18].

Si l'on néglige les recombinaisons radiatives, les électrons excités dans la deuxième sous-bande doivent d'abord relaxer vers la première sous-bande avec un moment $k_{\|}$important, puis il y a refroidissement du plasma de porteurs dans la sous-bande fondamentale. Ceci est illustré dans la figure 6. Comme le refroidissement de porteurs à l'intérieur de la même sous-bande est connu pour être très rapide $(\approx 150 \mathrm{fs})$, le temps de vie de la deuxième sous-bande est essentiellement gouverné par la relaxation inter sous-bande. Le temps mesuré de l'ordre de 10 ps suggère qu'il s'agit d'une relaxation par phonons optiques longitudinaux de grand

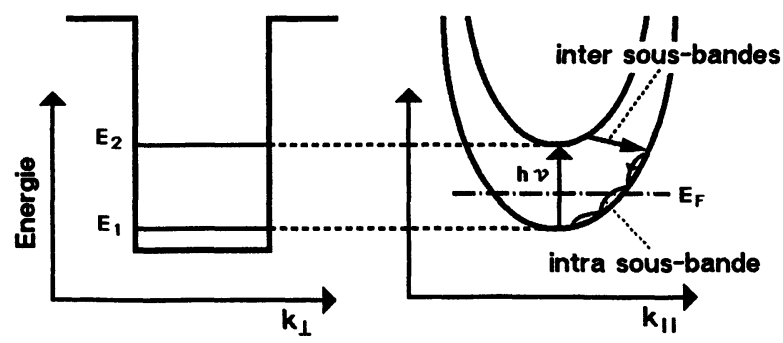

Fig. 6. - Représentation schématique des mécanismes de relaxation intrabande. Les schémas de gauche et de droite correspondent respectivement aux directions perpendiculaire et parallèle aux plans des couches du puits quantique. Les porteurs relaxent de la sous-bande excitée vers la sousbande fondamentale par émission d'un phonon optique longitudinal pour peu que la séparation énergétique $E_{2}-E_{1}$ le permette. Le refroidissement du plasma de porteurs par collisions dans la sous-bande fondamentale est très rapide.

[Intersubband relaxation processes. Left (right) scheme is related to the direction parallel (perpendicular) to the growth axis. Carrier relaxation from the excited subband to the ground subband involves a longitudinal optical phonon. Cooling of the carrier plasma within the ground subband is very rapid.] 
moment $[19,20]$. Notons que dans des puits quantiques de plus grande largeur $(240 \AA)$, la relaxation prédominante s'effectue par phonons acoustiques avec des temps de vie mesurés de l'ordre de $100 \mathrm{ps}$ [21].

\section{Conclusion.}

Un important travail de recherches est actuellement poursuivi à l'échelle internationale sur les transitions optiques dans la bande de conduction des puits quantiques AlGaAs. Outre qu'ils permettent une connaissance plus fine de la physique des puits quantiques, ces travaux devraient déboucher sur une nouvelle génération de photodétecteurs rapides infrarouges mais aussi sur de nouveaux dispositifs non linéaires. L'étude présentée ici concerne deux structures à puits quantiques à dopage modulé et anti-modulé. La transition d'absorption intrabande tombe dans les deux cas à une longueur d'onde proche de $10 \mu \mathrm{m}$. Les expériences utilisant un laser $\mathrm{CO}_{2}$ impulsionnel montre une saturation de l'absorption intrabande caractérisée par une intensité de saturation de l'ordre de $300 \mathrm{~kW} / \mathrm{cm}^{2}$. Un modèle simple nous a permis de déduire de ces valeurs un temps de vie des sous-bandes de la bande de conduction de l'ordre de $10 \mathrm{ps}$. Ces temps sont caractéristiques d'une relaxation par phonons optiques longitudinaux.

\section{Remerciements}

Le groupe de l'IEF souhaite remercier T. A. DeTemple et G. Bastard. Le groupe du LCR remercie E. Barbier, J. P. Hirtz pour la croissance de la structure à puits quantiques multiples.

\section{Bibliographie}

[1] Pinczuk, A., Stömer, H. L., Dingle, R., Worlock, J. M., WiegmanN, W., Gossard, A. C., Observation of intersubband excitations in a multilayer two-dimensional electron gas, Solid State Commun 32 (1979) 1001-1003.

[2] Pinczuk, A., Worlock, J. M., Light scattering by two-dimensional electron systems in semiconductors, Surf. Sci. 113 (1982) 69-84.

[3] Abstreiter, G., Merlin, R., Pinczuk, A., Inelastic light scattering by electronic excitations in semiconductors heterostructures, IEEE J. Quant. Electron. 22 (1986) 1771-1784.

[4] West, L. C., Eglash, S. J., First observation of an extremely large dipole infrared transition within the conduction band of a GaAs quantum well, Appl. Phys. Lett 46 (1985) 1156-1158.

[5] Karazinov, R. F., Suris, R. A., Possibility of the amplification of electromagnetic waves in a semiconductor with a superlattice, Sov. Phys.-Semicond. 5 (1971) 707-709.

[6] Harwit, A., HaRris, J. S., Observation of Stark shifts in quantum well intersubband transitions, Appl. Phys. Lett. 50 (1987) 685-687.

[7] Levine, B. F., Malik, R. J., Walker, J., Choi, K. K., Bethea, C. G., KleinmanN, D. A., VANDERBERG, J. M., Strong $8.2 \mu \mathrm{m}$ infrared intersubband absorption in doped GaAs/AlAs quantum well waveguides, Appl. Phys. Lett. 50 (1987) 273.

[8] Levine, B. F., Choi, K. K., BetheA, C. G., WALKER, J., MALIK, R. J., New $10 \mu \mathrm{m}$ infrared detector using intersubband absorption in resonant tunneling GaAlAs superlattices, Appl. Phys. Lett. 50 (1987) 1092-1094.

[9] Kastalsky, A., Duffield, T., Allen, S. J., HarbiSON, J., Photovoltaic detection of infrared light in a GaAs/AlGaAs superlattice, Appl. Phys. lett. 52 (1988) 1320-1322.
[10] Levine, B. F., Cho, A. Y., Walker, J., Malik, R. J., Kleinman, D. A., Sivco, D. L., InGaAs/InAlAs multiquantum well intersubband absorption at a wavelength of $\lambda=4.4 \mu \mathrm{m}$, Appl. Phys. Lett. 52 (1988) 1481-1483.

[11] Fröhlich, D., Wille, R., Schlapp, W., Weimann, G., Optical quantum Stark effect in GaAs quantum wells, Phys. Rev. Lett. 59 (1987) 1749-1751.

[12] QING, Y., CHU-Liang, Y., The effect of complex band structure and non-parabolicity on subbands of $\mathrm{GaAs}-\mathrm{Al}_{x} \mathrm{Ga}_{1-x}$ As heterostructures : band offset, J. Phys. C: Solid State Phys. 20 (1987) 5125-5134, et références à l'intérieur.

[13] Nakayama, N., Kuwahara, H., Kato, H., KUBOTA, K., Intersubband transitions in $\mathrm{GaAs}-\mathrm{Al}_{x} \mathrm{Ga}_{1-x} \mathrm{As}$ modulation-doped superlattices, Appl. Phys. Lett. 51 (1987) 1741-1743.

[14] BASTARD, G., communication privée.

[15] Newson, D. J., Kurobe, A., Possibility of optical bistability due to resonant intersubband excitation in stepped modulation-doped quantum wells, Appl. Phys. Lett. 51 (1987) 1670-1672.

[16] Ando, T., Fowler, A. B., Stern, F., Electronic properties of two-dimensional systems, Rev. Mod. Phys. 54 (1982) 437-672.

[17] Ahn, D., Chuang, S. L., Non-linear intersubband optical absorption in a semiconductor quantum well, J. Appl. Phys. 62 (1987) 3052-3054.

[18] Seilmeier, A., Hübner, H.-J., Abstreiter, G., WEIMANN, G., SCHLAPP, W., Intersubband relaxation in $\mathrm{GaAs}-\mathrm{Al}_{x} \mathrm{Ga}_{1_{-}} \mathrm{As}$ quantum well structures observed directly by an infrared bleaching technique, Phys. Rev. Lett. 59 (1987) 13451348.

[19] SHAH, J., Hot carriers in quasi 2-D polar semiconductors, IEEE J. Quantum Electron. 22 (1986) 17281743 , et références à l'intérieur. 
[20] Lugli, P., GoodNICK, S. M., Nonequilibrium Longitudinal-Optical Phonon Effects in GaAsAlGaAs Quantum Wells, Phys. Rev. Lett. 59 (1987), 716-719, et références à l'intérieur.
[21] Oberli, D. Y., WaKe, D. R., Klein, M. V., Klem, J., Henderson, T., Morkoç, H., Time-resolved Raman scattering in GaAs quantum wells, Phys. Rev. Lett. 59 (1987) 696-699. 